\title{
BMJ Open Evaluation of an OPEN Stewardship generated feedback intervention to improve antibiotic prescribing among primary care veterinarians in Ontario, Canada and Israel: protocol for evaluating usability and an interrupted time-series analysis
}

To cite: Acharya KR, Brankston G, Soucy J-PR, et al. Evaluation of an OPEN Stewardship generated feedback intervention to improve antibiotic prescribing among primary care veterinarians in Ontario, Canada and Israel: protocol for evaluating usability and an interrupted timeseries analysis. BMJ Open 2021;11:e039760. doi:10.1136/ bmjopen-2020-039760

- Prepublication history for this paper is available online. To view these files, please visit the journal online (http://dx.doi. org/10.1136/bmjopen-2020039760).

Received 24 April 2020 Revised 27 November 2020 Accepted 15 December 2020

\section{Linked}

- http://dx.doi.org/10.1136/ bmjopen-2020-039810

Check for updates

(C) Author(s) (or their employer(s)) 2021. Re-use permitted under CC BY-NC. No commercial re-use. See rights and permissions. Published by BMJ.

For numbered affiliations see end of article.

Correspondence to

Dr Amy L Greer;

agreer@uoguelph.ca

\section{ABSTRACT}

Introduction Antimicrobial resistance (AMR) impacts the health and well-being of animals, affects animal owners both socially and economically, and contributes to AMR at the human and environmental interface. The overuse and/or inappropriate use of antibiotics in animals has been identified as one of the most important drivers of the development of AMR in animals. Effective antibiotic stewardship interventions such as feedback can be adopted in veterinary practices to improve antibiotic prescribing. However, the provision of dedicated financial and technical resources to implement such systems are challenging. The newly developed web-based Online Platform for Expanding Antibiotic Stewardship (OPEN Stewardship) platform aims to automate the generation of feedback reports and facilitate wider adoption of antibiotic stewardship. This paper describes a protocol to evaluate the usability and usefulness of a feedback intervention among veterinarians and assess its impact on individual antibiotic prescribing.

Methods and analysis Approximately 80 veterinarians from Ontario, Canada and 60 veterinarians from Israel will be voluntarily enrolled in a controlled interrupted time-series study and their monthly antibiotic prescribing data accessed. The study intervention consists of targeted feedback reports generated using the OPEN Stewardship platform. After a 3-month preintervention period, a cohort of veterinarians (treatment cohort, $n=120$ ) will receive three feedback reports over the course of 6 months while the remainder of the veterinarians $(n=20)$ will be the control cohort. A survey will be administered among the treatment cohort after each feedback cycle to assess the usability and usefulness of various elements of the feedback report. A multilevel negative-binomial regression analysis of the preintervention and postintervention antibiotic

\section{Strengths and limitations of this study}

- Multinational pragmatic study.

The first study of its kind in a veterinary setting.

- Scalable and open concept with potential for broad generalisability to various care settings.

- The sample size could not be determined in the absence of the required parameters and as a result, the study may be underpowered.

- Differences in recruitment strategy for intervention groups across the two study sites may result in heterogeneity of intervention effect.

prescribing of the treatment cohort will be performed to evaluate the impact of the intervention.

Ethics and dissemination Research ethics board approval was obtained at each participating site prior to the recruitment of the veterinarians. The study findings will be disseminated through open-access scientific publications, stakeholder networks and national/ international meetings.

\section{INTRODUCTION}

\section{Burden of antibiotic resistance}

The anthropogenic development (selection) and dissemination (exchange across sectors) of antimicrobial resistant (AMR) organisms has become one of the biggest threats to human and animal health. AMR can adversely impact the health and welfare of animals and result in social and financial consequences for their owners. ${ }^{1}$ Antibiotic resistance has been identified as one of the greatest threats to poverty reduction, quality 
of life, health and survival in humans. ${ }^{1-3}$ National and international surveillance data show that the proportion of isolates resistant to commonly used antibiotics exceeds $50 \%$ in some settings ${ }^{45}$ contributing to poorer health outcomes and higher healthcare costs. ${ }^{5}$ It is estimated that by the year 2050, 10 million human deaths annually will be attributable to AMR with an associated global cost of US $\$ 100$ trillion. ${ }^{6}$ Antibiotic resistance that develops in animals and humans can be transmitted between species through various direct and indirect routes, ${ }^{7-9}$ necessitating a One Health approach for AMR control.

\section{Contributors to AMR: a focus on animal level contributors}

Among the anthropogenic contributors of AMR, ${ }^{10-14}$ the use and misuse of antibiotics in animals and humans has been clearly identified as the most important. ${ }^{815}$ Global antibiotic use has continued to increase by $65 \%$ between 2000 and 2015, partially driven by higher use in low and middle income countries. ${ }^{16}$ The rate of antibiotic use in animals is expected to escalate in some of these countries and is fuelled by a growth in agricultural industries in response to the growing demand for foods of animal origin. ${ }^{17}$ While the use of antibiotics in animals is a necessity in many cases, there are several scenarios which lead to the misuse of antibiotics. First, antibiotics (including those that are critically important for human medicine) are used non-therapeutically for growth promotion, or for an extended period of time for preventive purposes in many countries. ${ }^{18}$ Second, there is a high rate of misuse of antibiotics in countries that have poor veterinary control of antibiotic use. ${ }^{18}$ Third, the quality of the available antibiotics in some countries can be substandard or counterfeit ${ }^{19-21}$ due to weak quality control and quality assurance mechanisms in those countries. ${ }^{18}$ These practices are of global concern as AMR developed in one part of the world has the potential to rapidly transmit on a global scale. Given the limited number of new antibiotics available without pre-existing cross-resistance to the prevailing repertoire of antibiotics, it is imperative to preserve the effectiveness of the available antibiotics by reducing their unnecessary use. ${ }^{22}{ }^{23}$ Indeed, reducing antibiotic use has been associated with a $15 \%-32 \%$ reduction in the prevalence of antibiotic resistant bacteria. ${ }^{24}$ Recognising the multisectoral nature of the problem, a global reduction in antibiotic use can only be achieved by developing and adopting strategies on a global scale with application in both animal and human health sectors.

\section{Antibiotic stewardship}

Antibiotic stewardship refers to the concerted use of interventions to ensure the appropriate use of antibiotics so that their effectiveness is preserved, and public health is protected..$^{25}$ Stewardship measures have been initiated globally particularly in the human health sector to reduce the use of antibiotics in an effort to reduce the development and hence prevalence of antibiotic resistant bacteria. ${ }^{24}{ }^{26}$ This approach has been recognised as an effective way to reduce the overuse of antibiotics ${ }^{27}{ }^{28}$ by improving antibiotic prescribing, decreasing the duration of antibiotic use and reducing the use of antibiotics among physicians. ${ }^{29-31}$ Of the prescribing and enabling measures that have been used to achieve positive behavioural changes, audit and feedback approaches have been identified as important strategies and have been widely used by various stakeholders ${ }^{32} 33$ to improve the prescribing practices of medical professionals, ${ }^{32}$ usually in combination with other interventions. ${ }^{34}$ Such strategies could potentially be adopted in a veterinary setting to reduce the use of antibiotics in animals thereby promoting not only the health and well-being of the animals but also significantly contributing to the health and well-being of humans and the environment.

\section{Antibiotic stewardship and challenges for adoption in a veterinary setting}

The concept of antibiotic stewardship remains a relatively new concept in veterinary medicine, although historically there have been instances when the availability and use of medically important antibiotics in animals were limited with an associated reduction in the prevalence of AMR infections in humans. ${ }^{35-37}$ Recently, a global consensus to phase out the use of all classes of antibiotics in animals for growth promotion purposes has garnered notable progress. ${ }^{38}$ Theoretically, a veterinarian can commit to antibiotic stewardship by making a conscious effort to use antibiotics appropriately and when necessary, ${ }^{39}$ however, for the institutionalisation and successful operation of antibiotic stewardship in a veterinary setting, core activities should include accessing evidence-based prescribing guidelines as well as tracking, evaluating and reporting on antibiotic prescribing. ${ }^{40}$ Fulfilling these commitments can be challenging in a veterinary setting as essential resources might be lacking. For example, antibiotic prescription guidelines may be either unavailable or not current, the availability of stewardship tools that have been tested in a veterinary setting may be limited, and resources for the prompt laboratory-based diagnosis of an animal may be limited. ${ }^{38}$ Furthermore, the diversity of veterinary practices due to the level of care and specialisation they provide, and the species focus (companion animal, food animal and mixed practices) may make the design of universal stewardship interventions challenging. Finally, veterinary practices tend to have a small profit margin and, while various cost control strategies are implemented to ensure the profitability of a practice, the addition of antibiotic stewardship activities will incur additional costs. ${ }^{414}$ Thus, dedicating the funds and technical resources that would be required to operationalise antibiotic stewardship activities would be more challenging for small independent clinics compared with the practices that operate as larger groups. Innovative strategies and tools are thus required to facilitate the operationalisation of antibiotic stewardship in a veterinary setting. The automation of stewardship activities that have been successful in human medicine ${ }^{32} 33$ such as audit and feedback reports coupled with up-to-date best 
practice guidelines, would address the challenge posed by the diversity, size and scarcity of financial and technical resources in veterinary clinics and promote more widespread adoption of antibiotic stewardship initiatives in this sector.

\section{Online Platform for Expanding Antibiotic Stewardship as a tool} to address the challenge: a one health approach

In an effort to minimise these barriers, our team is conducting a multinational antibiotic stewardship initiative to promote responsible antibiotic prescribing in human and veterinary medicine, ${ }^{43}$ a One Health approach. The Online Platform for Expanding Antibiotic Stewardship (OPEN Stewardship) project uses a feedback intervention that combines local antibiotic prescription guidelines with feedback reports. The platform automates the process of analysing the prescriptions of individual practitioners and generating a feedback report based on their prescription records while also comparing individual data to the prescribing patterns of other practitioners. In addition, relevant antibiotic prescription guidelines will be incorporated into each feedback report. The open nature of the platform aims to reduce the financial barriers of antibiotic stewardship. The automation of the feedback mechanism addresses technical barriers to the implementation of antibiotic stewardship initiatives. Including prescribing guidelines will ensure that practitioners have access to the most current, evidence-based prescribing information that is relevant to their practice. Finally, evaluating this antibiotic stewardship initiative within a veterinary context will provide veterinarians with validated tools that are applicable to the veterinary medicine setting. The evaluation of the antibiotic stewardship will also be conducted among medical practitioners, the protocol for which will be published concurrently.

\section{The study approach and objectives}

In this pilot study, we will enrol primary care veterinarians in both Canada and Israel on a voluntary basis. The intervention group will obtain three feedback reports over a period of 6 months. After each feedback report, a survey will be administered to the intervention cohort of veterinarians to evaluate the various elements of the feedback report (intervention) for their usability and usefulness with the aim of improving the report structure and content before rolling the system out for broader use by the veterinary community. An interrupted time-series approach will be taken to compare the preintervention and postintervention change in the prescribing of the veterinarians in the intervention group to that of a comparable group of veterinarians in the non-intervention group. Although no microbiological evidence will be obtained to verify the appropriateness of an antibiotic prescription, we expect that the change in overall prescribing would be an appropriate proxy for the effect of the intervention. Likewise, we expect that most of the antibiotics used in noncomplicated upper respiratory infections in companion animals and mastitis in dairy cattle when the causative organism has not been identified and their antimicrobial sensitivity result not known are deemed inappropriate and unnecessary as a large proportion of the mastitis milk can be negative on culture ${ }^{44}$ and antibiotic treatment failure can be high depending on the causative organism, antibiotic resistance of the pathogen, age of animal and the chronicity of mastitis. ${ }^{45} 46$

Thus, it is expected that there would be a positive study outcome with regard to reduction in the amount of antibiotic use in animals following the intervention, similar to what has been observed among human physicians. ${ }^{47}$

\section{Objectives}

For a cohort of veterinarians in Ontario, Canada and Israel, to determine:

1. The usability and usefulness of each component of the feedback report, as well as the overall usefulness of the report.

2. The impact of the stewardship intervention (feedback report) on the overall antibiotic prescribing rate.

3. The impact of the stewardship intervention (feedback report) on the critically important antibiotic prescribing rate.

4. The impact of the stewardship intervention (feedback report) on the mean duration of antibiotic prescribing.

5. The impact of the stewardship intervention (feedback report) on the broad-spectrum antibiotic prescribing rate.

\section{METHODS AND ANALYSIS}

In this non-randomised study (figure 1), veterinarians from two countries (Canada and Israel) will be voluntarily enrolled in order to evaluate the usability and usefulness of individualised feedback reports and to assess the impact of feedback reports on the antibiotic prescribing of the veterinarians. In brief, after 3 months of enrolment in the study, a cohort of veterinarians will receive the intervention in the form of three feedback reports delivered electronically over a period of 6 months. Within 1 week of the receipt of the feedback report, a survey will be administered to evaluate its usability and usefulness among the veterinarians. The participants are required to receive all three feedback reports and respond to all three questionnaire surveys to be a part of the study.

\section{Study participants}

The participants of this study will be 140 veterinarians from two study regions: Ontario, Canada and Israel. The study will focus on veterinarians working in a general practice clinic in an outpatient setting. In Canada, 20 veterinarians with predominant dairy cattle practice and 60 veterinarians with predominant companion animal practice will be voluntarily enrolled from a pool of veterinarians holding a membership with the Ontario Association of Bovine Practitioners (OABP), or the Ontario Veterinary Medical Association (OVMA) respectively. In Canada, an email request containing the information 

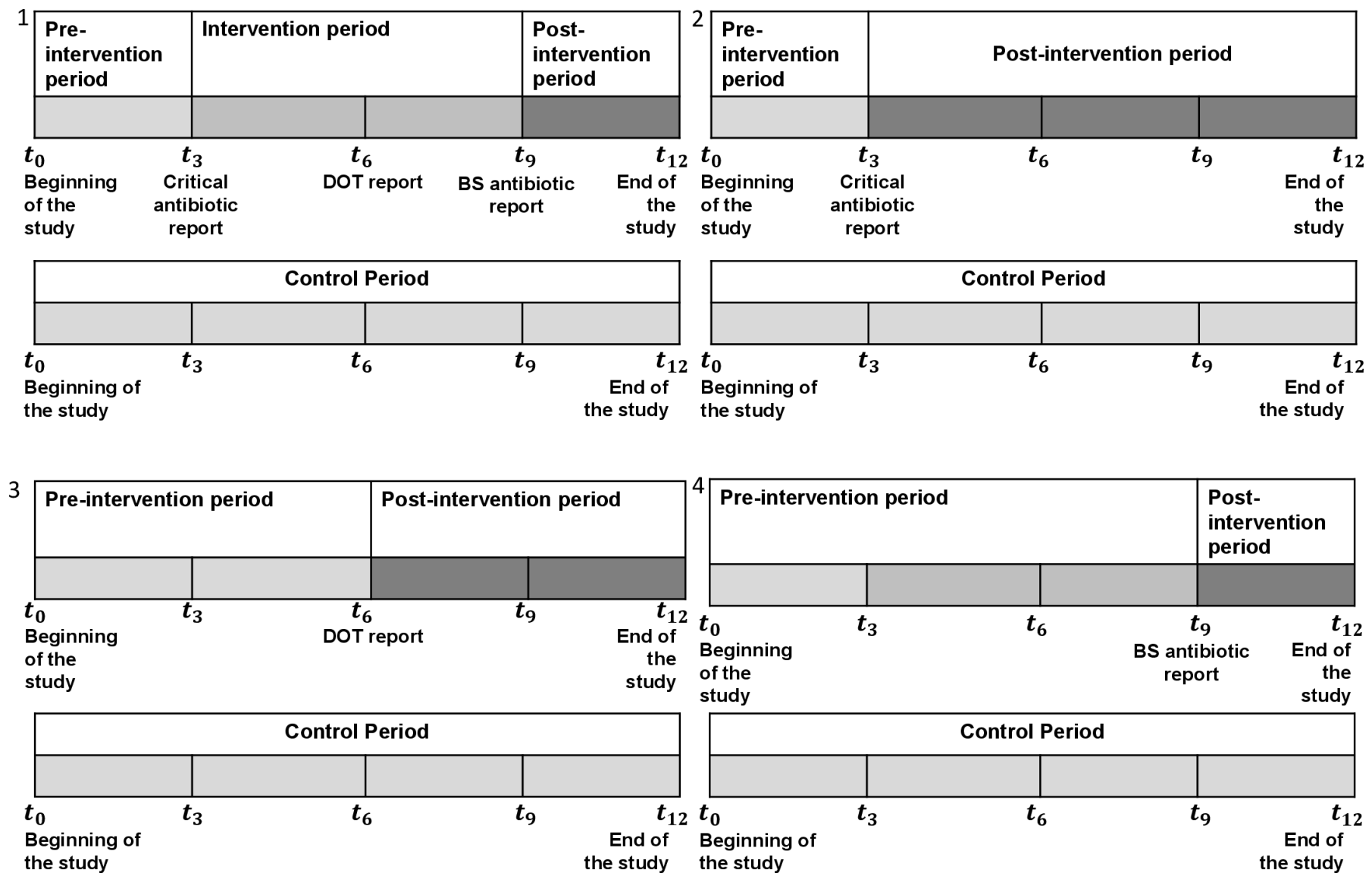

Figure 1 Timeline of the study. Block 1-4 depicts preintervention and postintervention periods corresponding to various analysis scenarios to realise objectives $2-5$, respectively, dot: duration of therapy, and $T_{0}$ to $T_{12}$ represent months since the beginning of the study.

regarding the study will be sent to the listserv of the OABP and the OVMA. A follow-up email will be sent if the sample size is not fulfilled. In Israel, 60 veterinarians with companion animal practice will be voluntarily enrolled from randomly selected small animal clinics. An email request containing the information regarding the study will be sent to randomly selected clinics in Israel. The information sent to the participants have details on the project background and rational so that the participants can understand the concept of antimicrobial stewardship. Likewise, we expect that some of the participants in Canada will already be familiar to the concept of antimicrobial stewardship due to the ongoing national discussions in the area of antimicrobial stewardship.

Veterinarians will be assigned to either the intervention cohort $(n=120)$ or the control cohort $(n=20)$. Control cohorts will be matched to the intervention cohorts by type of practice and postal code of the practice. Practitioners from within a single practice will not be assigned to both control and intervention cohorts. The intervention cohort of veterinarians will receive the intervention, whereas the rest will not receive the intervention (control cohort).

\section{Intervention}

The intervention is the quarterly delivery of three emails containing a personalised feedback report automatically generated using the OPEN Stewardship platform. For this, the antibiotic prescribing data of the participants will be extracted from the electronic practice management system of the participant following a protocol customised for each of the system. Once uploaded to the platform the data will be automatically analysed and feedback reports generated. The platform is also capable of automatically emailing the feedback report to the participants, however, during the study this feature in the platform will be disabled for privacy reasons. Each of the reports consist of a chart that compares the antibiotic prescribing of the veterinarian to the mean and the 25th percentile of the prescribing among other study participants in the same research location (eg, Canada or Israel) and a corresponding practice guideline. These reports will be sent over the period of 6 months at 3 months intervals. The timeline of the intervention has been provided in figure 1 and details on the composition of the interventions at each time point has been summarised in table 1. The level of prescribing details that will be obtained in this study is expected to be heterogeneous. Therefore, we decided to focus on overall prescribing without specifying the disease conditions. However, for the third report, the veterinarians will be requested to tag their antibiotic prescribing to a specific disease condition and hence the report will focus on the use of broad-spectrum antibiotics for these tagged diseases. 
Table 1 Composition of the feedback report at each time point

\begin{tabular}{|c|c|c|c|}
\hline Feedback reports & Reports & Benchmarking & Guidelines \\
\hline First feedback at $t_{3}$ & $\begin{array}{l}\text { 1. Overall rate of antibiotic use broken down by } \\
\text { class of antibiotics (number of prescriptions } \\
\text { of antibiotics prescribed per } 100 \text { cases) } \\
\text { (antimicrobials categorised by World Health } \\
\text { Organization (WHO) based on their importance to } \\
\text { human use).* }\end{array}$ & $\begin{array}{l}\text { Average across all } \\
\text { participating veterinarians } \\
\text { and the } 25 \text { th percentile from } \\
\text { each study site. }\end{array}$ & $\begin{array}{l}\text { Restricting the use of } \\
\text { antibiotic classes that are } \\
\text { critical for human use. }\end{array}$ \\
\hline Third feedback at $t_{9}$ & $\begin{array}{l}\text { 1. Rate of broad-spectrum antibiotic prescribing } \\
\text { 2. Average antibiotic prescription rate } \\
\text { 3. Overall rate of antibiotic use broken down by } \\
\text { class of antibiotics (antimicrobials categorised } \\
\text { by WHO based on their importance to human } \\
\text { use).* }\end{array}$ & $\begin{array}{l}\text { Average across all } \\
\text { veterinarians and the 25th } \\
\text { percentile. }\end{array}$ & $\begin{array}{l}\text { Reduce the use of broad- } \\
\text { spectrum antibiotics }\end{array}$ \\
\hline
\end{tabular}

*WHO list of Critically Important Antimicrobials for Human Medicine (WHO CIA list). 2019. Available from: https://www.who.int/ foodsafety/publications/antimicrobials-sixth/en/.

CIA, critically important antimicrobial.

\section{The first report (critical antibiotic report)}

This report will focus on the prescribing rate (number of prescriptions of antibiotics prescribed per 100 cases) for antibiotics that are considered critical for human medicine, also called critically important antimicrobials . While the feedback report will use simple metrics that are more intuitive, the time-series analysis will use more robust but less intuitive measures based on defined daily doses of antibiotics prescribed per 100 cases. ${ }^{48} 49$ Each personalised report will contain a comparison of the prescribing rate of the target veterinarian to the mean and the 25th percentile of the prescribing rate among other study participants in the same geographic location (figure 2). In addition, a clinical guideline regarding the restriction of critically important antibiotics for human medicine will also be provided which is based on the recommendation of the WHO and the World Organization for Animal Health (OIE).$^{50}{ }^{51}$ It is known that in companion animals, antibiotics that are considered critical for human medicine are prescribed to animals presenting with acute conditions like respiratory illness and the majority of antibiotic treatment in this setting is unnecessary. ${ }^{46}{ }^{51}$ Likewise, in dairy cattle, mastitis is the most frequent reason for antibiotic prescribing, whereby antibiotics are given without the identification of the causative pathogen, ${ }^{52}$ and given to the herd as a part of blanket dry cow therapy, while a majority of the formulations used are critically important for human medicine. ${ }^{515354}$ Thus, it would be a valid expectation of the stewardship intervention to achieve a reduction in the use of antibiotics critical for human medicine in both companion animals and dairy cattle.

\section{The second report (duration of therapy report)}

This report will focus on the average duration of antibiotic prescribing. For many disease conditions in both companion animals and dairy cattle, the recommended duration of therapy is less than 7 days. The desirable outcome of the feedback interventions would be to reduce the number of prescriptions written for a duration of 7 days or greater. The information on the duration of therapy is not expected to be always present in the medical records of the participants. In this situation, this will be indirectly calculated. Likewise, we will consult with the prescriber to identify if an on-farm treatment protocol is present and if available the protocol will be used to determine the duration of therapy in dairy cattle. The guideline that will be included in this report will be derived from the available guidelines ${ }^{55} 56$ and from the recommended average duration of therapy of the registered products.

\section{The third report (broad-spectrum antibiotic report)}

This report will focus on the rate of broad-spectrum antibiotic prescribing. The rationale of this report is to reduce the unnecessary use of broad-spectrum antibiotics for disease conditions when the etiological agent has not been identified, as happens in the case of diseases like upper respiratory tract infections in companion animals and mastitis in dairy cattle. While some of the broadspectrum antibiotics can also be critically important antibiotics, the merit of this feedback lies in providing feedback to the veterinarians on their antibiotic prescribing for a specific disease condition.

Objective 1: usability and usefulness of the feedback report A longitudinal study will be conducted among the intervention cohort via administration of online questionnaires 


\section{Your prescribing of critically important antibiotics compared to other participants of this study}

Your prescribing of antibiotics considered "Critically Important" was $42 \%$ more than average prescribing of the participants of this study and you are in the highest $25 \%$ of the prescribers. Although in total you prescribed less than the average prescribing of the participants of this study, you total prescribing was higher than lower $25 \%$ of the prescribers.

Number of antibiotic prescriptions per 100 client-contact

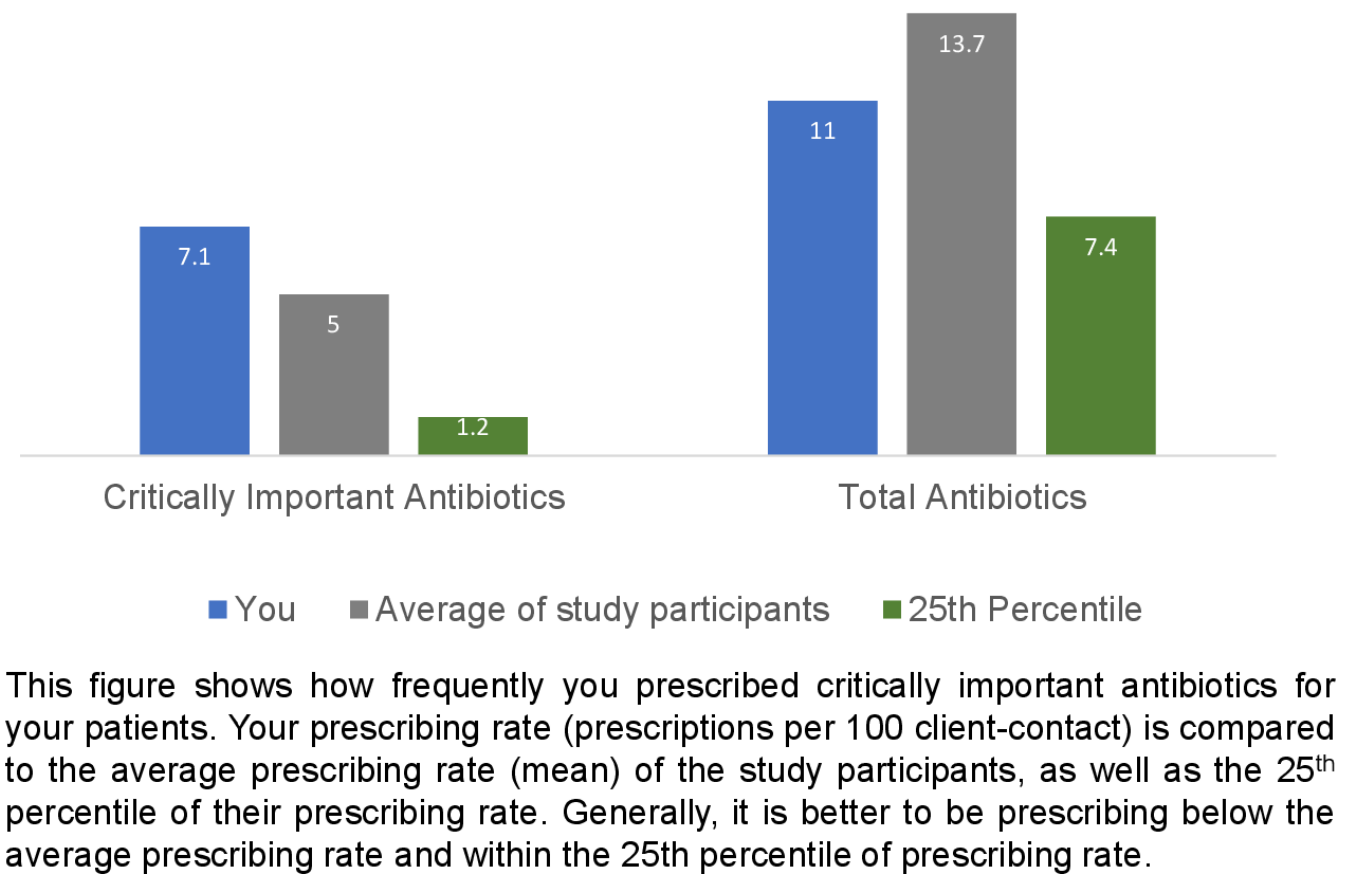

Figure 2 An example chart which will be a component of the feedback report on critically important antibiotic prescribing.

to evaluate the feedback reports generated by the OPEN Stewardship platform. The study will evaluate both the usability and the usefulness of the feedback report, with an emphasis on improving reports for the future use of this tool. ${ }^{57}$ The rationale behind choosing a longitudinal study design is to reduce common method variance bias which is more pronounced when using a single response from participants at a single time point. ${ }^{58}$ The use of feedback reports as an antibiotic stewardship tool for veterinarians has not been previously evaluated so following enrolled veterinarians over time allows participants to become accustomed to the reports thereby allowing for the identification of their true perceptions on the usability and usefulness of the tool. In total, three questionnaires will be administered within a week of receiving each of the three feedback reports spaced 3 months apart. Although longitudinal studies can have respondent attrition as a potential drawback, in this study that is less of a concern as the veterinarians will be associated with the study for a year and the survey will be conducted during the middle of the study (figure 1). The participants are required to receive three feedback reports and respond to three questionnaire surveys to be a part of the study.

\section{Questionnaires}

The survey questionnaires have been developed by the Public Health Agency of Sweden in consultation with all the study collaborators. Hebrew versions of the survey will be made available to the study participants in Israel. All three questionnaires will focus on the usability and usefulness of the various elements of the report (table 2). Additionally, the final questionnaire will seek to understand the future usability of the various elements of the report among the participants and to identify if the participants see the value of the report for their peers (an additional usability measure). Likewise, we will also attempt to identify the optimal frequency of the feedback reports (a usability measure). The questionnaires have been piloted among a test group of veterinarians at both study locations with an aim to validate the content, to identify and address issues associated with the questions and interpretation, and to estimate an average response time to 


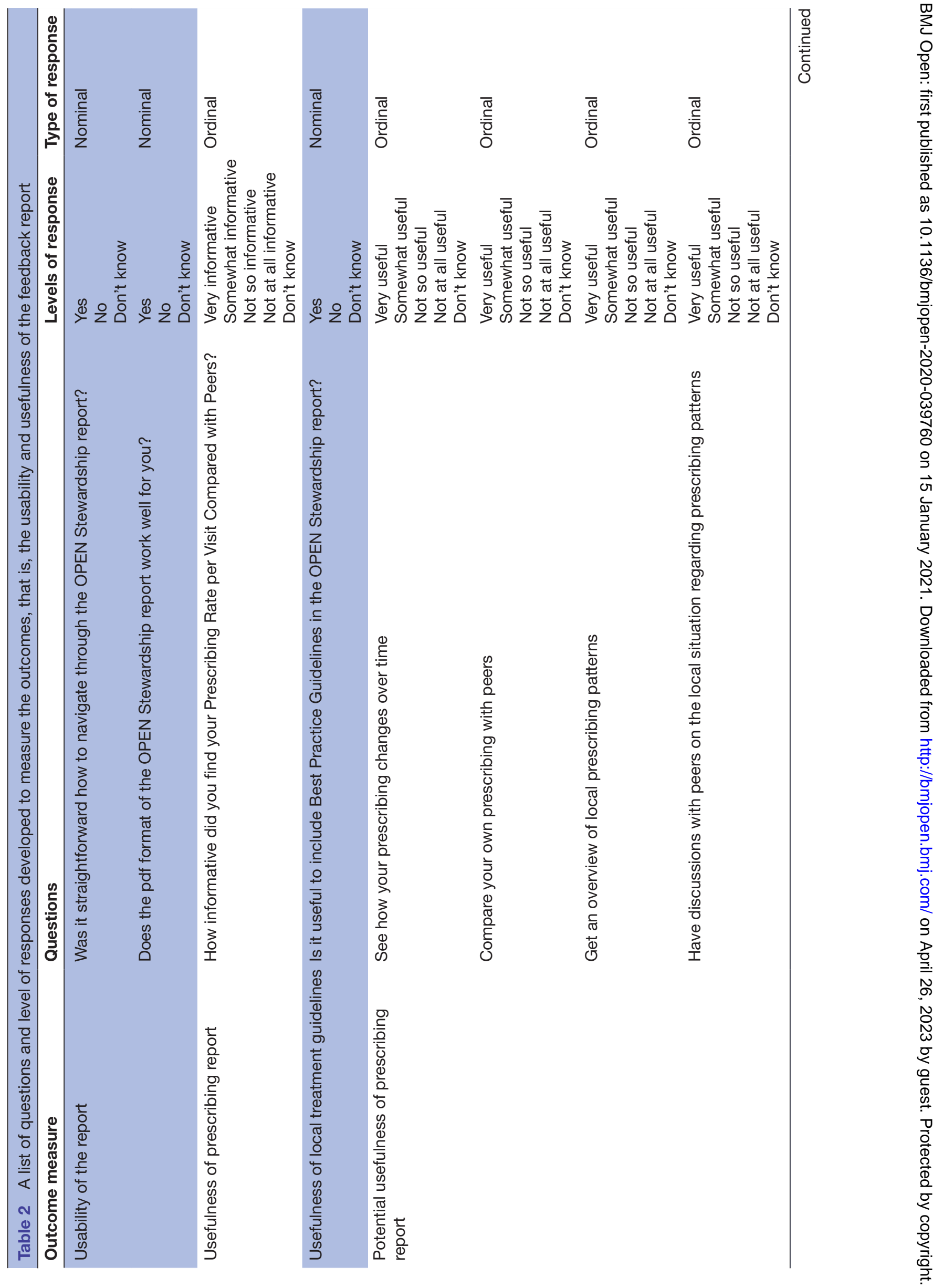




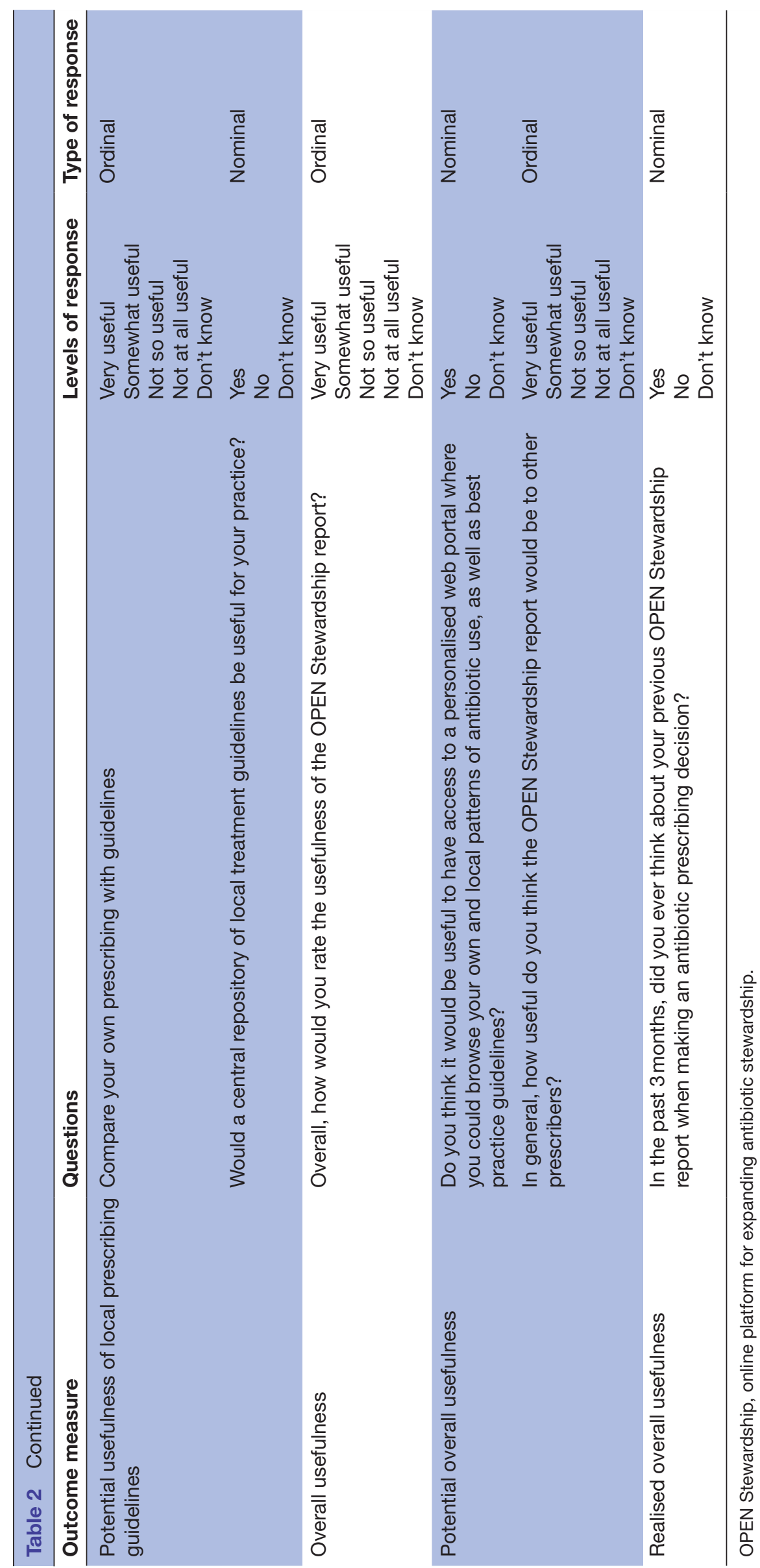

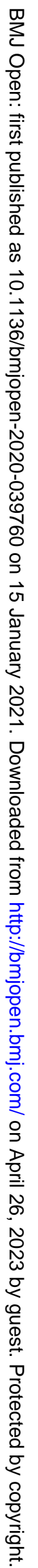


complete the survey. The detailed protocol for piloting the questionnaire survey will be published separately (reference to human protocol).

\section{Sample size}

The sample size for the survey includes the intervention cohort of the study $(n=120)$.

\section{Survey administration}

Three online surveys will be administered among the participants via the Qualtrics platform (Qualtrics, Provo, Utah, USA). A link to the survey will be emailed to the intervention cohort of study participants within a week of receipt of each feedback report.

\section{Outcomes}

The outcomes of the study will be the usability of the feedback report, usefulness of each component of the feedback report and overall usefulness of the feedback report as a categorical (nominal or ordinal) variable. The outcomes on the Likert scale will be treated as ordinal variables for data analysis. These outcomes will be measured by means of several questions as summarised in table 2. The questions evaluating the practical aspect of receiving, opening and navigating through the feedback report will aid us in understanding the usability of the feedback report. Whereas, the questions assessing the value of each component of the feedback report and the overall report to the veterinarians will help us to understand the usefulness of the feedback report. The questions have been designed to understand both perceived and actual usefulness of the feedback report.

\section{Covariates}

The predictors of interest include covariates such as study location (Canada vs Israel), gender (male vs female) of the veterinarian, employment status (full time or part time), type of practice (single clinic vs multiclinic) and composition of patients (by species).

\section{Data analysis}

Descriptive statistics will be used to understand the usability and usefulness of the feedback report generated by the OPEN Stewardship platform. Exploratory data analysis will be conducted to identify potential determinants of the perceived usability and usefulness of the feedback report. Mean scores and variance at each time point will be calculated and analysis of variance will be used to assess if the usability and usefulness of the feedback report changed over time. Ordinal and or multinomial regression analysis will be performed to evaluate a relationship between the outcome variable and covariates such as type of veterinary clinic, study location and gender of the veterinarians.

\section{Objectives 2-5: impact of the intervention on antibiotic prescribing patterns}

We will use a quasi-experimental interrupted time-series design to evaluate the impact of the intervention on antibiotic prescribing patterns. Interrupted time series is widely used to evaluate public health interventions and has been identified as an alternative to randomised trials when there are ethical or practical obstacles to conducting randomised controlled trials. ${ }^{59}$ Interrupted time-series studies have been rarely used in veterinary care settings to evaluate interventions. ${ }^{60}$ This study is the first attempt to evaluate an antibiotic stewardship intervention in a veterinary setting. An additional strength of the study includes the inclusion of participants from two locations and the provision of external controls.

A multilevel negative binomial regression approach will be taken to assess the impact of the intervention, that is, change in level and trend of antibiotic prescribing of the veterinarians. ${ }^{61}$ This will be performed by comparing the preintervention and postintervention antibiotic prescribing of the intervention cohorts, and by comparing the prescribing of intervention cohorts with that of control cohorts.

\section{Sample size and power estimation}

The sample size for evaluating the impact of intervention on antibiotic prescribing includes both the intervention $(n=120)$ and control $(n=20)$ cohort of this study.

The antibiotic prescribing rates will be modelled as negative binomial rates. So, the sample size estimation approach would be the one applicable for comparing two negative binomial rates. ${ }^{62}$ While the approach requires a value for the common dispersion parameter, in the absence of historical antibiotic stewardship intervention studies in veterinary setting, a reasonable estimate of the common dispersion parameter is not possible. Likewise, it is not currently possible to estimate the antibiotic prescribing rate of a veterinarian as we have no reference to the baseline antibiotic prescribing rate among veterinarians that is comparable to our research setting. It is also difficult to estimate the average duration of exposure, the dispersion or over-dispersion contributed by differences in case flows contributed by seasonality and at the individual level, practice level, and country level, and/ or the relative effect size the intervention would produce that are required for estimating sample size. ${ }^{62}$ Thus, in this pilot study, sample size was determined heuristically. We have however attempted to include two study locations, a relatively large sample size, and numerous data points over time. The presence of external controls are an additional strength of the study, the absence of which has been identified as a limitation in similar studies conducted among human physicians. ${ }^{63}$ Although it is not logical to estimate the power of the study based on the sample size of the study, it is expected that the study will likely be underpowered. However, this study is unique to a veterinary setting and may provide a basis to calculate sample sizes for future studies.

\section{Outcomes}

The primary outcome of the interrupted time-series portion of the study is the monthly antibiotic prescribing 
rate (number of defined daily doses of antibiotics prescribed per 100 cases). The defined daily doses of antimicrobials used for dairy cattle will be calculated according to Lardé et $\mathrm{al}^{49}$ while a modification of this approach will be used to calculate the defined daily dose for antibiotics used in companion animals. The secondary outcomes of the study are (1) the rate of antibiotic prescribing for drug classes deemed critically important for human medicine (number of defined daily doses of critically important antibiotics prescribed per 100 cases), (2) the mean duration of antibiotic prescribing, and (3) the rate of prescribing broad-spectrum antibiotics (number of defined daily doses of broad-spectrum antibiotics prescribed per 100 cases) for a specific condition (eg, mastitis in cattle, and acute respiratory tract infection in companion animals).

Since participation in the study is voluntary, it is possible that the veterinarians who agree to participate in the study may have had previous experience with stewardship interventions resulting in less significant reductions in prescribing over time. Likewise, antibiotic stewardship is a new concept in veterinary clinics in Canada and Israel, a difference in level of exposure of the participants to antibiotic stewardship at the two study sites will be insignificant. However, a heterogeneity in the effect of the intervention in the two study locations is expected.

\section{Covariates}

Other potential predictors of interest include prescriber age, gender, employment status (full-time, or part-time), and composition of patients (by species, age, sex, and production status in dairy). The covariates will be tested for confounding and the confounding covariates that are biologically plausible will be included in the regression model. Likewise, the interaction of the covariates with study site will be included and assessed.

Additionally, random intercepts for prescribers, practices, and region will be fit to account for the prescriber level, practice level and regional heterogeneity in prescribing patterns. Seasonality will be accounted for by categorising the month variable. In addition, residual autocorrelation will also be considered during analysis. The covariates are summarised in table 3 .

\section{Statistical analysis}

We will use multilevel negative binomial regression models to analyse the controlled interrupted time-series data as these models can better explain the change in antibiotic prescribing rate due to stewardship interventions with the postintervention period compared with that of the preintervention period. ${ }^{6465}$ A series of multivariable regression analyses will be conducted as outlined in table 4 to address the specific research objectives.

The intervention effect will be modelled as a change in level and slope after administration of the feedback

\begin{tabular}{|c|c|c|c|}
\hline Variable & Description & Form & Role \\
\hline Region & ID of region (Israel orOntario, Canada) & ID & Random intercept \\
\hline Type of veterinary clinic & Multiclinic practice vs single clinic practice & Categorical & Fixed effect \\
\hline Veterinarian & ID of veterinarians & $\begin{array}{l}\text { ID (nested within practice } \\
\text { group) }\end{array}$ & Random intercept \\
\hline Age of the veterinarian & Age of the veterinarian when they enrol in the study & Integer & Covariate \\
\hline Sex of the veterinarian & Male vs female & Binary & Covariate \\
\hline $\begin{array}{l}\text { Employment status of } \\
\text { veterinarian }\end{array}$ & Part time vs full time employment of the veterinarian & Binary & Covariate \\
\hline $\begin{array}{l}\text { Experience of the } \\
\text { veterinarian }\end{array}$ & Years of practice of veterinarian & Integer & Covariate \\
\hline Seasonality & Month & Categorical & Random slope \\
\hline $\begin{array}{l}\text { Veterinarian } \\
\text { characteristics }\end{array}$ & Age, sex, and employment status (part time or full time) & Categorical & Fixed effect \\
\hline Patient composition & $\begin{array}{l}\text { Age group (percentage of animals that are up to } 1 \text { year } \\
\text { and percentage of animals that are adult), species } \\
\text { (percentage of patients that are canine vs feline vs } \\
\text { bovine), sex (percentage of patients that are female), } \\
\text { and production stage of dairy cattle (lactating cow vs } \\
\text { dry cow) }\end{array}$ & Categorical & Fixed effect \\
\hline
\end{tabular}

$\mathrm{ID}=$ Identity 
Table 4 Various analysis scenarios to fulfil objectives two to five of the study

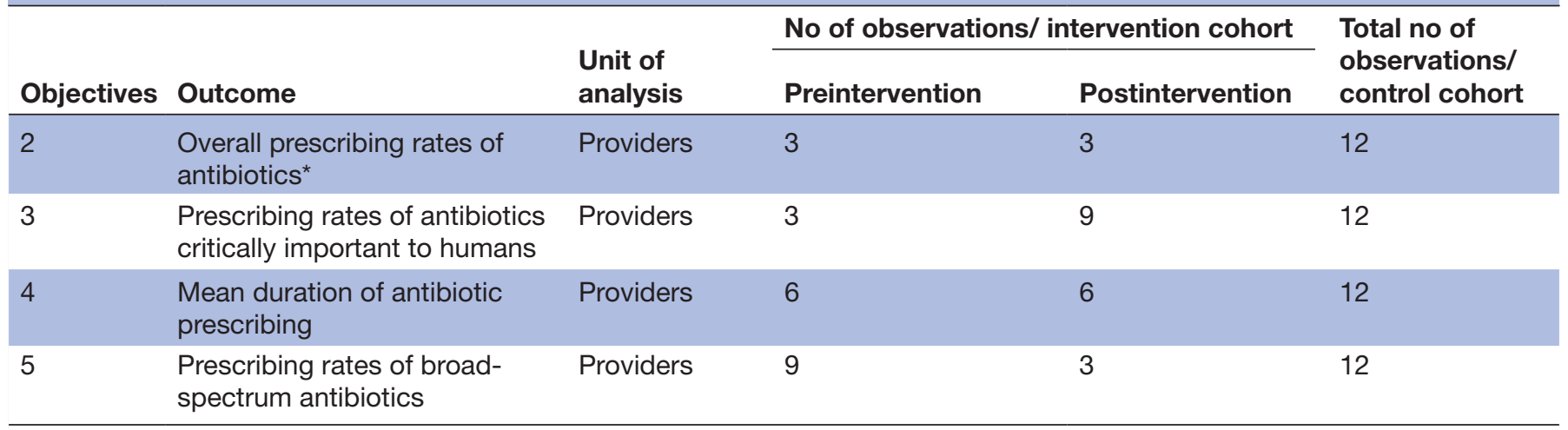

The change in preintervention period, intervention period and postintervention period reflect the order in which the three feedback reports (intervention) are provided to the participants

${ }^{*}$ The overall prescribing rate will be influenced by all three feedback reports (intervention). Hence the postintervention data points for overall prescribing rate will be the last 3 months following the receipt of the last feedback report.

intervention except for the analysis using overall antibiotic prescribing rates which will be modelled as a change in level and slope after the intervention period. A random slope will be used to account for the heterogeneity of effect among each veterinarian and across the two countries in our study. The postintervention period will be different for each of the regression models as shown in figure 1 and table 4 . The antibiotic prescribing of the veterinarians in the intervention cohort will also be compared with the prescribing of the control cohorts to examine the potential effects of other concurrent interventions on the antibiotic prescribing of the veterinarians.

\section{Autocorrelation and seasonality}

Autocorrelation in the outcome is expected in timeseries data. Autocorrelation will be handled by including random intercepts in the regression model and by controlling seasonality. Seasonality will be controlled by stratification of the outcome by month. ${ }^{61}$

\section{Sensitivity analyses}

Alternative parameterisation of the study outcomes will be considered. An alternative outcome that may be considered is the prescribing rate of the most common antibiotics that are known to be specifically prescribed for mastitis in dairy cows or upper respiratory tract infections in companion animals. Additionally, the outcome will be stratified by age, species, sex and subsequent analysis will be conducted. Likewise, we will assess the real impact of the intervention by evaluating some proxy outcomes like the rate of contact with the clients.

\section{Ethics and dissemination}

Research ethics board (REB) approval has been obtained from the institutional REB at each study site in Canada (University of Guelph REB \#19-03-006), and in Israel (Internal Research Review Committee of the Koret School of Veterinary Medicine - Veterinary Teaching Hospital \#KSVM-VTH/05_2019) prior to the enrolment of the participants and acquisition of the prescription data. A guideline will be provided to the participant so that they can anonymise and aggregate the prescription data by month before making them available to the researchers. The data will be stored in an encrypted manner on a secure server and data accessibility will be restricted to the researcher team. The study participants from Ontario, Canada will be compensated with a gift card, whereas the participants in Israel will not be compensated.

The results of this study will be disseminated through the stakeholder networks of the institutions involved in this study. Likewise, we will publish the research findings in open access, peer-reviewed journals.

\section{Stakeholder involvement}

Veterinarians at both study locations were consulted during the design of this study and piloting of the survey.

Author affiliations

${ }^{1}$ Department of Population Medicine, University of Guelph, Guelph, Ontario, Canada ${ }^{2}$ Division of Epidemiology, Dalla Lana School of Public Health, University of Toronto, Toronto, Ontario, Canada

${ }^{3}$ Koret School of Veterinary Medicine, The Robert H. Smith Faculty of Agriculture, Food and Environment, The Hebrew University of Jerusalem, Rehovot, Israel

${ }^{4}$ Public Health Agency of Sweden, Stockholm, Sweden

${ }^{5}$ School of Public Health, Faculty of Health Sciences, Ben-Gurion University of the Negev, Beer-Sheva, Israel

${ }^{6}$ McMaster Health Forum, McMaster University, Hamilton, Ontario, Canada ${ }^{7}$ Department of Health Systems Management, Guilford Glazer Faculty of Business and Management and Faculty of Health Sciences, Ben-Gurion University of the Negev, Beer Sheva, Israel

${ }^{8}$ Institute for Health Policy, Management and Evaluation, University of Toronto, Toronto, Ontario, Canada

${ }^{9}$ Ottawa Hospital Research Institute, Ottawa, Ontario, Canada

Correction notice This article has been corrected since it first published. The provenance and peer review statement has been included.

Contributors KRA, GB, J-PRS, AC, AH, SL, ND, ME, DNF, JM-G, AS, DRM and ALG conceived the study and analysis. KRA codrafted the protocol with GB and ALG and will conduct the data analysis, J-PRS contributed to the refinement of the methodology section of the protocol, AC wrote the Israel specific part of the methodology section, $\mathrm{AH}$ and SL drafted the questionnaire and piloting protocol for the questionnaire and all the coauthors reviewed the questionnaire. All the authors reviewed the protocol and agree to the final version being submitted. 
Funding This work was supported by the funding from the Canadian Institutes of Health Research (CIHR), under the European Commission's Joint Programming Initiative on Antimicrobial Resistance (5th Joint Call).

\section{Competing interests None declared.}

Patient and public involvement Patients and/or the public were not involved in the design, or conduct, or reporting, or dissemination plans of this research.

Patient consent for publication Not required.

Provenance and peer review Not commissioned; externally peer reviewed.

Open access This is an open access article distributed in accordance with the Creative Commons Attribution Non Commercial (CC BY-NC 4.0) license, which permits others to distribute, remix, adapt, build upon this work non-commercially, and license their derivative works on different terms, provided the original work is properly cited, appropriate credit is given, any changes made indicated, and the use is non-commercial. See: http://creativecommons.org/licenses/by-nc/4.0/.

\section{ORCID iDs}

Kamal Raj Acharya http://orcid.org/0000-0001-6707-3536

Jean-Paul R Soucy http://orcid.org/0000-0002-8422-2326

Derek R MacFadden http://orcid.org/0000-0002-3838-1211

\section{REFERENCES}

1 The World Bank. Drug-Resistant infections: a threat to our economic future, 2019. Available: https://www.worldbank.org/en/topic/health/ publication/drug-resistant-infections-a-threat-to-our-economic-future

2 UNICEF. Time is running out - AMR Insights, 2019. Available: https:// www.amr-insights.eu/time-is-running-out/

3 WHO. Antibiotic resistance, 2019. Available: http://www.fao.org/3/ a-bc310e.pdf

4 PHAC. Canadian antimicrobial resistance surveillance system update 2018. Ottawa, 2018. Available: https://www.canada.ca/content/dam/ phac-aspc/documents/services/publications/drugs-health-products/ canadian-antimicrobial-resistance-surveillance-system-2018-reportexecutive-summary/pub1-eng.pdf

5 World Health Organization. Antimicrobial resistance: global report on surveillance. WHO: Geneva, Switzerland, 2014

6 O'Neil J. Review on antimicrobial resistance antimicrobial resistance: tackling a crisis for the health and wealth of nations. London: Review on Antimicrobial Resistance, 2014.

7 Price LB, Stegger M, Hasman H, et al. Staphylococcus aureus CC398: host adaptation and emergence of methicillin resistance in livestock. mBio 2012;3:1-6.

8 Holmes AH, Moore LSP, Sundsfjord A, et al. Understanding the mechanisms and drivers of antimicrobial resistance. Lancet 2016:387:176-87.

9 Robinson TP, Wertheim HFL, Kakkar M, et al. Animal production and antimicrobial resistance in the clinic. Lancet 2016:387:e1-3.

10 Stockwell VO, Duffy B. Use of antibiotics in plant agriculture. Rev Sci Tech 2012;31:199-210.

11 McManus PS, Stockwell VO, Sundin GW, et al. Antibiotic use in plant agriculture. Annu Rev Phytopathol 2002;40:443-65.

12 Larsson DGJ, de Pedro C, Paxeus N. Effluent from drug manufactures contains extremely high levels of pharmaceuticals. $J$ Hazard Mater 2007;148:751-5.

13 Ji X, Shen Q, Liu F, et al. Antibiotic resistance gene abundances associated with antibiotics and heavy metals in animal manures and agricultural soils adjacent to feedlots in Shanghai; China. $J$ Hazard Mater 2012:235-236:178-85.

14 Diwan V, Tamhankar AJ, Khandal RK, et al. Antibiotics and antibioticresistant bacteria in waters associated with a hospital in Ujjain, India BMC Public Health 2010;10:414.

15 IACG. No time to wait: securing the future from drug-resistant infections, 2019. Available: https://www.who.int/antimicrobialresistance/interagency-coordination-group/IACG_final_report_EN. pdf?ua $=1$

16 Klein EY, Van Boeckel TP, Martinez EM, et al. Global increase and geographic convergence in antibiotic consumption between 2000 and 2015. Proc Natl Acad Sci U S A 2018;115:E3463-70.

17 Van Boeckel TP, Brower C, Gilbert M, et al. Global trends in antimicrobial use in food animals. Proc Natl Acad Sci U S A 2015;112:5649-54.

18 World Organisation for Animal Health (OIE). OIE annual report on the use of antimicrobial agents intended for use in animals, 2018. Available: http://www.oie.int/fileadmin/Home/eng/Our_scientific_ expertise/docs/pdf/AMR/Annual_Report_AMR_3.pdf
19 Cockburn R, Newton PN, Agyarko EK, et al. The global threat of counterfeit drugs: why industry and governments must communicate the dangers. PLoS Med 2005;2:e100-8.

20 World Economic Forum. How fake animal medicines threaten African livestock, 2019. Available: https://www.weforum.org/agenda/2015/ 02/how-fake-animal-medicines-threaten-african-livestock/

21 Buckley GJ, Gostin LO. Countering the problem of falsified and substandard drugs. Washington, DC: National Academies Press, 2013.

22 Theuretzbacher U, Gottwalt S, Beyer P, et al. Analysis of the clinical antibacterial and antituberculosis pipeline. Lancet Infect Dis 2019;19:e40-50.

23 Butler MS, Blaskovich MA, Cooper MA. Antibiotics in the clinical pipeline at the end of 2015. J Antibiot 2017;70:3-24.

24 Tang KL, Caffrey NP, Nóbrega DB, et al. Restricting the use of antibiotics in food-producing animals and its associations with antibiotic resistance in food-producing animals and human beings: a systematic review and meta-analysis. Lancet Planet Health 2017:1:e316-27.

25 Pan-Canadian Public Health Network. Antimicrobial stewardship, 2016. Available: http://www.phn-rsp.ca/pubs/anstew-gestan/indexeng.php\#fnb5

26 Mölstad S, Löfmark S, Carlin K, et al. Lessons learnt during 20 years of the Swedish strategic programme against antibiotic resistance. Bull World Health Organ 2017;95:764-73.

27 Davey P, Brown E, Charani E. Interventions to improve antibiotic prescribing practices for hospital inpatients. In: Davey P, ed. Cochrane database of systematic reviews. Chichester, UK: John Wiley \& Sons, Ltd, 2013: CD003543.

28 Drekonja DM, Filice GA, Greer N, et al. Antimicrobial stewardship in outpatient settings: a systematic review. Infect Control Hosp Epidemiol 2015;36:142-52.

29 Gould IM. Benefits of antimicrobial stewardship in hospitals: evidence from a recent cochrane review. J Microbiol Immunol Infect 2015;48:S24

30 Davey P, Brown E, Charani E, et al. Interventions to improve antibiotic prescribing practices for hospital inpatients. Cochrane Database Syst Rev 2013:CD003543.

31 Davey P, Brown E, Charani E, Marwick CA, Scott CL, et al. Interventions to improve antibiotic prescribing practices for hospital inpatients. Cochrane Database Syst Rev 2013:CD003543.

32 Ivers N, Jamtvedt G, Flottorp S, et al. Audit and feedback: effects on professional practice and healthcare outcomes. Cochrane Database Syst Rev 2012;2012:CD000259.

33 Chung GW, Wu JE, Yeo CL, et al. Antimicrobial stewardship: a review of prospective audit and feedback systems and an objective evaluation of outcomes. Virulence 2013;4:151-7.

34 Grimshaw JM, Shirran L, Thomas R, et al. Changing provider behavior: an overview of systematic reviews of interventions. Med Care 2001;39:II2-45.

35 Wierup M. The Swedish experience of the 1986 year ban of antimicrobial growth promoters, with special reference to animal health, disease prevention, productivity, and usage of antimicrobials. Microbial Drug Resistance 2001;7:183-90.

36 Aarestrup FM, Seyfarth AM, Emborg HD, et al. Effect of abolishment of the use of antimicrobial agents for growth promotion on occurrence of antimicrobial resistance in fecal enterococci from food animals in Denmark. Antimicrob Agents Chemother 2001;45:2054-9.

37 Dutil L, Irwin R, Finley R, et al. Ceftiofur resistance in Salmonella enterica serovar Heidelberg from chicken meat and humans, Canada. Emerg Infect Dis 2010;16:48-54.

38 World Organization for Animal Health (OIE). Resolution No. 36 combating antimicrobial resistance through a one health approach: actions and OIE strategy. Paris: World Organization for Animal Health (OIE), 2016.

39 Anon. Key elements for implementing antimicrobial stewardship plans in bovine veterinary practices working with beef and dairy operations, 2017. Available: www.ebvma.org

40 Sanchez GV, Fleming-Dutra KE, Roberts RM, et al. Core elements of outpatient antibiotic stewardship. MMWR Recomm Rep 2016;65:1-12.

41 Hardefeldt LY, Gilkerson JR, Billman-Jacobe H, et al. Barriers to and enablers of implementing antimicrobial stewardship programs in veterinary practices. J Vet Intern Med 2018;32:1092-9.

42 Doherty C. Reaching new heights: results of the 2018 CVMA practice owners economic survey. Can Vet $J$ 2019;60:315-8.

43 Hulth A, Löfmark S, Andre J, et al. A tool for promoting responsible antibiotic prescribing across settings and sectors. Online $J$ Public Health Inform 2019;11. 
44 Makovec JA, Ruegg PL. Results of milk samples submitted for microbiological examination in Wisconsin from 1994 to 2001. J Dairy Sci 2003;86:3466-72.

45 Barkema HW, Schukken YH, Zadoks RN. Invited review: the role of cow, pathogen, and treatment regimen in the therapeutic success of bovine Staphylococcus aureus mastitis. J Dairy Sci 2006;89:1877-95.

46 Murphy CP, Reid-Smith RJ, Boerlin P, et al. Out-patient antimicrobial drug use in dogs and cats for new disease events from community companion animal practices in Ontario. Can Vet $J$ 2012;53:291-8.

47 Meeker D, Linder JA, Fox CR, et al. Effect of behavioral interventions on inappropriate antibiotic prescribing among primary care practices: a randomized clinical trial. JAMA 2016;315:562-70.

48 Redding LE, Muller BM, Szymczak JE. Small and large animal veterinarian perceptions of antimicrobial use metrics for hospitalbased stewardship in the United States. Front Vet Sci 2020;7:582.

49 Lardé H, Dufour S, Archambault M, et al. Assignment of Canadian defined daily doses and Canadian defined course doses for quantification of antimicrobial usage in cattle. Front Vet $\mathrm{Sci}$ 2020;7:10.

50 OIE. OIE list of antimicrobial agents of veterinary importance, 2019 Available: https://www.oie.int/fileadmin/Home/eng/Our_scientific expertise/docs/pdf/AMR/A_OIE_List_antimicrobials_July2019.pdf

51 WHO. WHO list of critically important antimicrobials for human medicine (who CIA list), 2019. Available: https://www.who.int/ foodsafety/publications/antimicrobials-sixth/en/

52 Oliveira L, Ruegg PL. Treatments of clinical mastitis occurring in cows on 51 large dairy herds in Wisconsin. J Dairy Sci 2014;97:5426-36.

53 Anonymous. Prescription drug list, 2019. Available: https://www. canada.ca/en/health-canada/services/drugs-health-products/drugproducts/prescription-drug-list/list.html\#a2

54 Saini V, McClure JT, Léger D, et al. Antimicrobial use on Canadian dairy farms. J Dairy Sci 2012;95:1209-21.
55 Weese JS, Blondeau J, Boothe D, et al. International Society for companion animal infectious diseases (ISCAID) guidelines for the diagnosis and management of bacterial urinary tract infections in dogs and cats. Vet $J$ 2019;247:8-25.

56 Lappin MR, Blondeau J, Boothe D, et al. Antimicrobial use guidelines for treatment of respiratory tract disease in dogs and cats: antimicrobial guidelines Working group of the Internationa Society for companion animal infectious diseases. $J$ Vet Intern Med 2017;31:279-94.

57 Keil M, Beranek PM, Konsynski BR. Usefulness and ease of use: field study evidence regarding task considerations. Decis Support Syst 1995;13:75-91.

58 Rindfleisch A, Malter AJ, Ganesan S, et al. Cross-sectional versus longitudinal survey research: concepts, findings, and guidelines. Journal of Marketing Research 2008;45:261-79.

59 Grimshaw J, Campbell M, Eccles M, et al. Experimental and quasi-experimental designs for evaluating guideline implementation strategies. Fam Pract 2000;17 Suppl 1:11S-16.

60 Ryu S, Lau CL, Chun BC. The impact of livestock manure control policy on human leptospirosis in Republic of Korea using interrupted time series analysis. Epidemiol Infect 2017;145:1320-5.

61 Bernal JL, Cummins S, Gasparrini A. Interrupted time series regression for the evaluation of public health interventions: a tutorial. Int J Epidemiol 2017;46:348-55.

62 Zhu H, Lakkis H. Sample size calculation for comparing two negative binomial rates. Stat Med 2014;33:376-87.

63 Allerberger F, Gareis R, Jindrák V, et al. Antibiotic stewardship implementation in the EU: the way forward. Expert Rev Anti Infect Ther 2009;7:1175-83.

64 Taljaard M, McKenzie JE, Ramsay CR, et al. The use of segmented regression in analysing interrupted time series studies: an example in pre-hospital ambulance care. Implement Sci 2014;9:77.

65 Gebski V, Ellingson K, Edwards J, et al. Modelling interrupted time series to evaluate prevention and control of infection in healthcare. Epidemiol Infect 2012;140:2131-41. 